\title{
The Application of Correlation Techniques to the Angular Spectrum of Scattered Radiation from Tokamak Plasmas ${ }^{1}$
}

\author{
R. Nazikian \\ Princeton Plasma Physics Laboratory \\ Princeton, NJ 08543
}

\begin{abstract}
Abatract
In the limit of the first Born approximation for a partially coherent secondary source, consisting of a spatially random plasma illuminated by a coherent plane wave, it is shown that the spectral coherence of the scattered radiation as measured on an arbitrary plane beyond the scatterer conveys information on the three dimensional intensity distribution of the random source. By defining a new two point statistical measure of the random field, closely related to the cross spectral density, we show that the fluctuation amplitude of the random source along the direction of the incident plane wave may be recovered from the measurement of the scattered radiation. The application of cross spectral techniques to fluctuation studies on tokamaks is considered.
\end{abstract}

1 


\section{Introduction}

The theory of partial coherence as developed by Wolf ${ }^{2}$ deals with the relationship between a planar radiation source and the spatial coherence of the radiated angular spectrum. It is only recently, however, that attention has turned to the three dimensional inverse scattering problem for random secondary anisotropic sources (i.e., coherent scattering from random refractive index fluctuations ${ }^{3}$ ). The key principle in the theory of partial coherence is that the state of coherence of the radiated angular spectrum carries information on the spatial distribution of the inhomogeneous radiator.

In standard scattering experiments on laboratory plasmas it is often assumed that only the spectral power density of the fluctuations, expressed in terms of the plasma form factor $S_{n}(K, \omega)$, contains useful information on the random medium. ${ }^{4}$ Unfortunately, the spectral power density alone contains no information on the spatial distribution of the raudom medium. As the poor spatial resolution of scattering systems for long wavelength fluctuations remains a major diagnostic limitation, it is appropriate to investigate whether the measurement of the angular coherence of the scattered radiation may be used to overcome this 
limitation.

\section{Spectral Correlation Analysis}

We first consider a plane wave $u_{0}=\exp \left(j k_{0} z\right)$ incident on a bounded plasma density distribution $n_{e}(\mathbf{r})$. Under the first Born approximation the scattered angular spectrum $A_{\mathbf{s}}\left(\mathbf{K}_{\perp} ; z\right)$ (the two dimensional spatial Fourier transform in $\mathbf{r}_{\perp}$ of the scattered field $\left.u_{s}\left(r_{\perp} ; z\right)\right)$ in the parabolic approximation is given by ${ }^{5}$

$$
\begin{aligned}
A_{s}\left(\mathbf{K}_{\perp} ; z\right) & =\boldsymbol{G}\left(\kappa_{F} ; z\right) N\left(\mathbf{K}_{F}\right) \\
\mathcal{G}\left(\kappa_{F} ; z\right) & =-1 / 2 j k_{0} \exp \left(j \kappa_{F} z\right)
\end{aligned}
$$

where for the purpose of this paper we consider $N\left(\mathbf{K}_{F}\right)$ the three dimensional spatial Fourier transform of the fluctuating part of the normalized plasma density $n(\mathbf{r})=\left(n_{e}(\mathbf{r})-\left\langle n_{e}(\mathbf{r})\right\}\right) / n_{c r}$, $n_{\sigma \tau}=k_{0}^{2} / 4 \pi r_{e}\left(r_{e}\right.$ is the classical electron radius), $\mathcal{G}$ is the propagator for the scattered radiation, $\kappa_{F}=\left(K_{\perp}, \kappa_{F}\right)$ is the wave vector of the scattered field and $\mathbf{K}_{F}=\left(\mathbf{K}_{\perp}, \mathrm{K}_{F}\right)$ is the corresponding wave vector in the plasma. To satisfy momentum conservation for the scattered field we require $K_{F}=\kappa_{F}-k_{0} \simeq$ $-K_{\perp}^{2} / 2 k_{0}$ in the parabolic approximation valid for forward an- 
gle scattering. Given that in near field experiments it is more convenient to measure the scattered complex phase $\psi_{s}\left(r_{\perp} ; z\right)$ where the radiated field $u=u_{0} \exp \left(\psi_{s}\right)$, the appropriate expression for the two dimensional Fourier transform $\Psi_{s}\left(K_{\perp} ; z\right)$ of the scattered complex phase in the first Rytov approxination is, ${ }^{5}$

$$
\begin{aligned}
& \Psi_{s}\left(\mathbf{K}_{\perp} ; z\right)=\mathcal{R}\left(K_{F} ; z\right) N\left(\mathbf{K}_{F}\right), \\
& \mathcal{R}\left(K_{F} ; z\right)=-1 / 2 j k_{0} \exp \left(j K_{F} z\right) .
\end{aligned}
$$

For plane wave illumination the angular spectrum $A_{\text {s }}$ and the phase spectrum $\Psi_{z}$ only differ by a constant phase for a fixed observer plane, hence for convenience, we deal for the most part with the complex phase. Given a sufficiently large set of independent observations of a homogeneous random medium, we may estimate the plasma form factor $S_{n}(\mathrm{~K})$ by taking ensemble averages over either $A_{g} A_{s}^{*}$ or $\Psi_{\mathfrak{s}} \Psi_{s}^{*}$, where

$$
\begin{aligned}
\left\langle\left|A_{s}\left(\mathbf{K}_{\perp} ; z\right)\right|^{2}\right\rangle=\left\langle\left|\Psi_{s}\left(\mathbf{K}_{\perp} ; z\right)\right|^{2}\right\rangle & =1 / 4 k_{0}^{2}\left\langle\left|N\left(\mathbf{K}_{F}\right)\right|^{2}\right\rangle \\
& =\lambda_{0}^{2} r_{e}^{2} \bar{n}_{e} V_{p} S_{n}\left(\mathbf{K}_{F}\right)
\end{aligned}
$$

where the plasma form factor is

$$
S_{n}\left(\mathbf{K}_{F}\right)=\left\langle\left|N_{e}\left(\mathbf{K}_{F}\right)\right|^{2}\right\rangle / \bar{n}_{e} V_{p}
$$


and where $\bar{n}_{e}$ and $V_{p}$ are the mean plasma density and plasma volume, respectively.

This result is very familiar and forms the basis for coherent scattering techniques on tokamak plasmas. However, the fluctuation volume is seldom known a priori and is usually assumed to be the intersection of the transmitter and receiver patterns with the plasma volume assuming the random medium is homogeneous. To obtain further information on the distribution of the fluctuations within the scattering volume, it is necessary to determine the coherence of the angular sphectrum of scattered radiation. In a generalization of the spectral density, the cross spectral density $W$, is defined as

$$
W_{\mathbf{s}}\left(\mathbf{K}_{\perp}, \mathbf{K}_{\perp}^{\prime} ; z\right)=\left\langle\Psi_{s}^{*}\left(\mathbf{K}_{\perp} ; z\right) \Psi_{s}\left(\mathbf{K}_{\perp}^{\prime} ; z\right)\right\rangle,
$$

which reduces to the usual spectral power density for $\mathbf{K}_{\perp}=$ $\mathbf{K}_{\perp}^{\prime}$. Also, given the two point density correlation function $C_{n}\left(\mathbf{r}_{1}, \mathbf{r}_{2}\right)=\left\langle n\left(\mathbf{r}_{1}\right) n\left(\mathbf{r}_{2}\right)\right\rangle$, we readily obtain the result,

$$
W_{s}\left(K_{\perp}, K_{\perp}^{\prime} ; z\right)=1 / 4 k_{0}^{2} \exp \left[j\left(K_{F}^{\prime}-K_{F}\right) z\right] C_{n}\left(-K_{F}, K_{F}^{\prime}\right)
$$

where $C_{n}\left(\mathbf{K}_{F}, \mathbf{K}_{F}^{\prime}\right)$ is the six dimensional Fourier transform of the spatial correlation function evaluated in the parabolic approximation on the surface $\mathrm{K}_{F}=\left(\mathrm{K}_{1}, K_{F}\right)$ in wavenumber 
space and where use is made of the Hermitian symmetry of the Fourier transform of the normalized density, $N^{*}(-\mathbf{K})=N(\mathbf{K})$. The coherence of the angular spectrum of scattered radiation is given by the normalized cross spectral density,

$$
\mu_{s}\left(\mathbf{K}_{\perp}, \mathbf{K}_{\perp}^{\prime} ; z\right)=\frac{W_{s}\left(\mathbf{K}_{\perp}, \mathbf{K}_{\perp}^{\prime} ; z\right)}{\left[\mathcal{W}_{s}\left(\mathbf{K}_{\perp}, \mathbf{K}_{\perp} ; z\right)\right]^{1 / 2}\left[\mathcal{W}_{s}\left(\mathbf{K}_{\perp}^{\prime}, \mathbf{K}_{\perp}^{\prime} ; z\right)\right]^{1 / 2}}
$$

where upon substitution of Eq. (8) into Eq. (9) we obtain the important relation

$$
\begin{array}{r}
\mu_{s}\left(\mathbf{K}_{\perp}, \mathbf{K}_{\perp}^{\prime} ; z\right)=\exp \left[j\left(K_{F}^{\prime}-K_{F}\right) z\right] \\
\times \frac{C_{n}\left(-\mathbf{K}_{F}, \mathbf{K}_{F}^{\prime}\right)}{\left[\mathbf{C}_{n}\left(-\mathbf{K}_{F}, \mathbf{K}_{F}\right)\right]^{1 / 2}\left[\mathcal{C}_{n}\left(-\mathbf{K}_{F}^{\prime}, \mathbf{K}_{F}^{\prime}\right)\right]^{1 / 2}} .
\end{array}
$$

The normalized cross spectral density has been studied at great length with respect to planar sources, however, for weak scattering from extended three dimensional ranciom media, we need to introduce a very useful new quantity, the cross spectral correlation,

$$
\tilde{W}_{s}\left(\mathbf{K}_{\perp}, \mathbf{K}_{\perp}^{\prime} ; z\right)=\left\langle\Psi_{s}\left(\mathbf{K}_{\perp} ; z\right) \Psi_{s}\left(\mathbf{K}_{\perp}^{\prime} ; z\right)\right\rangle
$$

and proceeding in a similar fashion as above, we may define the normalized cross spectral correlation of the field,

$$
\tilde{\mu}_{s}\left(\mathbf{K}_{\perp}, \mathbf{K}_{\perp}^{\prime} ; z\right)=\frac{\overline{\mathcal{W}}_{s}\left(\mathbf{K}_{\perp}, \mathbf{K}_{\perp}^{\prime} ; z\right)}{\left[\mathcal{W}_{\mathrm{o}}\left(\mathbf{K}_{\perp}, \mathbf{K}_{\perp} ; z\right)\right]^{1 / 2}\left[\mathcal{W}_{s}\left(\mathbf{K}_{\perp}^{\prime}, \mathbf{K}_{\perp}^{\prime} ; z\right)\right]^{1 / 2}}
$$


which in terms of the plasma two point density correlation becomes

$$
\begin{gathered}
\tilde{\mu}_{s}\left(\mathbf{K}_{\perp}, \mathbf{K}_{\perp}^{\prime} ; z\right)=-\exp \left[j\left(\mathrm{~K}_{F}+\mathrm{K}_{F}^{\prime}\right) z\right] \\
\times \frac{\mathcal{C}_{n}\left(\mathbf{K}_{F}, \mathbf{K}_{F}^{\prime}\right)}{\left[\mathcal{C}_{n}\left(-\mathbf{K}_{F}, \mathbf{K}_{F}\right)\right]^{1 / 2}\left[C_{n}\left(-\mathbf{K}_{F}^{\prime}, \mathbf{K}_{F}^{\prime}\right)\right]^{1 / 2}} .
\end{gathered}
$$

To this point we have only dealt with various old and new definitions. We now assume an analytic model for the two point density correlation $C_{n}$ in which the the mean square amplitude of fluctuations $\left\langle n^{2}\right\rangle$ varies slowly compared to the fluctuation correlation length. This is known as the quasi-homogeneous approximation introduced by Wolf, ${ }^{2}$

$$
C_{n}\left(\mathbf{r}, \mathbf{r}^{\prime}\right)=I\left(1 / 2\left(\mathbf{r}+\mathbf{r}^{\prime}\right)\right) \rho\left(\mathbf{r}-\mathbf{r}^{\prime}\right)
$$

where $I(\mathbf{r}) \simeq\left\langle n^{2}(\mathbf{r})\right\rangle$ and for a homogeneous medium, $I(\mathbf{r})$ is just a constant. It can then be shown that the six dimensional Fourier transform $C_{n}$ of $C_{n}$ may be expressed in the form

$$
C_{n}\left(\mathbf{K}, \mathbf{K}^{\prime}\right)=\Sigma\left(\mathbf{K}+\mathbf{K}^{\prime}\right) S\left(1 / 2\left(\mathbf{K}-\mathbf{K}^{\prime}\right)\right),
$$

where $\Sigma(\mathbf{K})$ is the Fourier transform of $I(\mathbf{r})$ and $S(\mathbf{K})$ is the Fourier transform of $\rho(\mathbf{r})$. To interpret this expression, we have for a homogeneous medium,

$$
C_{n}\left(\mathbf{K},-\mathbf{K}^{\prime}\right)=\delta\left(\mathbf{K}-\mathbf{K}^{\prime}\right) S\left(1 / 2\left(\mathbf{K}+\mathbf{K}^{\prime}\right)\right),
$$


which is simply proportional to the form factor for $\mathbf{K}=\mathbf{K}^{\prime}$, and is zero otherwise. We note that in the quasi-homogeneous approximation, the correlation length [functional width of $\rho(\mathbf{r})$ ] is considerably narrower than the scale length for mean square amplitude variation in the medium [variations in $I(\mathbf{r})$ ] and so by the nature of Fourier transforms, the function $S(K)$ is uniform over the region of spectral coherence determined by the function $\Sigma(\mathbf{K}) .^{6}$

Substituting Eq. (15) into Eq. (13) and evaiuating the normalized speciral correlation for opposite scattering vectors, $\mathbf{K}_{\boldsymbol{F}}=$ $\left(\mathbf{K}_{\perp}, K_{F}\right)$ and $\mathbf{K}_{F}^{\prime}=\left(-\mathbf{K}_{\perp}, K_{F}\right)$ (c.f. Fig. 1) we obtain,

$$
\begin{array}{r}
\tilde{\mu}_{s}\left(\mathbf{K}_{\perp},-\mathbf{K}_{\perp} ; z\right)=-\exp \left(j 2 K_{F} z\right) \\
\times \frac{\Sigma\left(0,2 K_{F}\right) S\left(\mathbf{K}_{\perp}, 0\right)}{\Sigma(0,0) S^{1 / 2}\left(\mathbf{K}_{\perp}, K_{F}\right) \mathcal{S}^{1 / 2}\left(\mathbf{K}_{\perp},-K_{F}\right)}
\end{array}
$$

where use is made of the symmetry of $S(\mathbf{K})$. By quasi-homogeveity and within the parabolic approximation, $\left|\mathbf{K}_{F}\right| \simeq\left|\mathbf{K}_{\perp}\right|$ and $S\left(\mathbf{K}_{\perp}, K_{F}\right) \simeq$ $S\left(\mathbf{K}_{\perp}, 0\right)$ for $\Sigma\left(0,2 K_{F}\right) / \Sigma(0,0) \sim 1$. Hence the normalized cross spectral correlation is approximately given by

$$
\tilde{\mu}_{\triangleleft}\left(\mathbf{K}_{\perp},-\mathbf{K}_{\perp} ; z\right)=-\exp \left(j 2 K_{F} z\right) \Sigma\left(2 K_{F}\right) / I_{0}
$$

where we have assumed the random medium is homogeneous in the direction perpendicular to the incident plane wave, (valid in 
narrow beam geometries) and where

$$
I_{0}=\int_{-\infty}^{\infty} I(z) d z
$$

The homogeneity condition for fluctuations perpendicular to the beam is not necessary, but it is convenient for the present analysis.

We now define a new function, the spectral coherence

$$
\hat{\mathbf{I}}\left(2 K_{F} ; z_{0}\right)=-\tilde{\mu}_{s}\left(\mathbf{K}_{\perp},-\mathbf{K}_{\perp} ; z_{0}\right)
$$

which is Hermitian symmetric in $K_{F}$ and where the measurement is performed on the plane $z=z_{0}$. Experimentally, the spectral coherence may be determined from the measured complex phase only for $K_{F}=-\left|K_{F}\right|$, however, as the form of Eq. (18) must be Hermitian symmetric in $K_{F}$ [due to the Hermitian symmetry of $\Sigma(\mathbf{K})$ ], we may simply extend the measured conerence into $K_{F}=+\left|K_{F}\right|$. We note at this point that apart from the normalizing factor $I_{0}, \hat{I}\left(K_{F} ; z_{0}\right)$ is in fact the Fourier transform of $I\left(z^{\prime}\right)$ where $z^{\prime}=z+z_{0}$. Multiplying $\hat{I}\left(K_{F} ; z_{0}\right)$ by the inverse Fourier kernel $\exp \left[j K_{F}\left(z-z_{0}\right)\right]$ and integrating over $K_{F}$ we obtain, (given $K_{0}$ is the highest resolved wavenumber in the plasma),

$$
\hat{I}(z)=\int_{-K_{0}^{2} / k_{0}}^{K_{0}^{2} / k_{0}} \exp \left[j K\left(z-z_{0}\right)\right] \hat{I}\left(K ; z_{0}\right) d K / 2 \pi
$$




$$
\begin{aligned}
& \simeq \int_{-\infty}^{\infty} \exp \left[j K\left(z-z_{0}\right)\right] \hat{I}\left(K ; z_{0}\right) d K / 2 \pi \\
& =I(z) / I_{0}
\end{aligned}
$$

provided $\hat{Y}\left(K_{F} ; z_{0}\right)$ is fully determined (i.e., for large enough $\left.K_{0}\right)$. The essential result is that by determining the normalized cross spectral correlation from the complex phase of the scattered radiation, both the absolute location of the scatterer as well as the intensity distribution of the secondary radiation source in the direction of beam propagation may be determined. Further, apart from the quasi-homogeneity constraint, the result obtained is independent of the degree of spatial isotropy of the scatterer. In comparison, for the plane wave illumination of a random refractive medium, the normalized cross spectral density $\mu_{0}$ of the scattered field provides little information on the distribution of the random medium in the direction of the incident wave.

For the integration in Eq. (21) to approximate an inverse Fourier transform, a condition on the minimum useful probing wavelength $\lambda_{0}$ must be satisfied. Namely, we require $K_{0}^{2} / k_{0} \geq \Delta \zeta_{z} \sim$ $2 \pi / L_{z}$ where $L_{z}$ is the thickness of the fluctuating region. Hence, we require $\lambda_{0} \geq \Lambda^{2} / L_{z}$ where $\Lambda$ is the shortest resolved wavelength. For fusion plasmas, taking $\Lambda=1 \mathrm{~cm}$ and $L_{z} \sim 1 \mathrm{~m}$, then 
the optimum wavelength $\lambda_{0}$ is around $100 \mu \mathrm{m}$. We point out that eron if the absolute phase of the scattered field for all wavenumbers cannot $k \in$ determined such as in standard microwavis scattering experiments, the normalized correlation $\left|\tilde{\mu}_{s}\right|$ may still be measured by heterodyne detection techniques commonly used in plasma fluctuation studies. It can be shown that for receivers sampling afsroximately the same wavenumber, the coherence hetween two detector signals is related to $\left|\mu_{s}\left(\mathbf{K}_{\perp}, \mathbf{K}_{\perp}^{\prime} ; z\right)\right|$ (the normaluzed cross spectral density), from whici the solid angle of the scatterer at the receivers may be determined. However, if the detecior signals sampling opposite wavevectors are correlated, the coherence is releted to the normalized spectral correlation $\mid \bar{\mu}_{s}\left(\mathbf{K}_{\perp},-\mathbf{K}_{\perp} ; \boldsymbol{z}\right)$ !

Finally, if we introduce a Gaussian envelope

$$
I(z)=n_{0} \exp \left(-\left(z-z_{1}\right)^{2} / L_{z}^{2}\right)
$$

over an otherwise homogeneous random medium, we then obtain for the normalized cross spectral correiation,

$$
\left\{\tilde{\mu}_{*}\left(\mathbf{K}_{\perp},-\mathbf{K}_{\perp} ; z\right) \mid=\exp \left(-Q^{2}\right)\right.
$$

where $Q=\left|K_{F}\right| L_{z}$ is simply the Klein-Cook parameter, ${ }^{7}$ a parameter derived elsewhere in the context of scattering from non- 
random sinusoidal phase screens of finite extent.

\section{Conclusion}

By the introduction of a new two point statistical measure over the angular spectrum of scattered radiation from random plesma fluctuations, the local intensity of the scatterer along the direction of the incident plane wave may be recovered. Experimentally, for large magnetically confined plasmas, a single beam interferometer operating at $\sim 100 \mu \mathrm{m}$ is sufficient to recover the local intensity of plasma fluctuations given that the quasihomogeneity constraint is a reasonable approximation to the plasma fluctuations.

\section{Acknowledgments}

I wish to acknowledge the efforts of Dr. John Howard for carefully reading the manuscript and for providing various suggestions. This work was supported by the U.S. Department of Energy Contract No. DE-AC02-76CH03073. 


\section{Bibliography}

[1] submitted to the Review of Scientific Instruments (1990).

[2] E. WOLF and W.H. CARTER, Angular Distribution of Radiant Intensity From Sources of Different Degrees of Spatial Coherence, Opties Communications. 13205 (1975).

[3] W.H. CARTER and E. WOLF, Scattering From QuasiHomogeneous Media, Optics Communications. 6785 (1988).

[4] E. HOLZHAUER and J.H. MASSIG, An Analysis of Optical Mixing in Plasma Scattering Experiments, Plasma Physics and Controlled Fusion.20 867 (1978).

[5] J. HOWARD, R. NAZIKIAN, and L.E. SHARP, Diffaction Tomography for Plasma Refractive Index Measurements, submitted to Plasma Physics and Controlled Fusion (1989).

[6] W.H. CARTER and E. WOLF, Coherence and Radiometry with Quasihomogeneous Planar Sources, J. Opt. Soc. Am. 67785 (1976).

[7] J. HOWARD, L.E. SHARP, and R. NAZIKIAN, $A$ Linear Systems Approach to Forward Angle Laser Scattering Diagnostics, Proceedings of the $4^{\text {th }}$ International Symposium on Laser-Aided Plasma Diagnostics, Fukuoka Japan (1989). 


\section{Figure Captions}

\section{Fig.1}

Scattered radiation from random plasma fluctuations is projected onto the Fourier transform plane of a lens. Correlations between various points in the Fourier transform plane may be used to recover the three dimensional mean square distribution of density fluctuations within the scattering volume. The mapping from spatial to spectral coordinates in the Fourier transform plane of the lens and within the parabolic approximation is given by $\mathbf{K}_{\perp}=\boldsymbol{r}_{\perp} k_{0} / f$ where $f$ is the focal length of the lens. ${ }^{5}$ 
\#90X0194

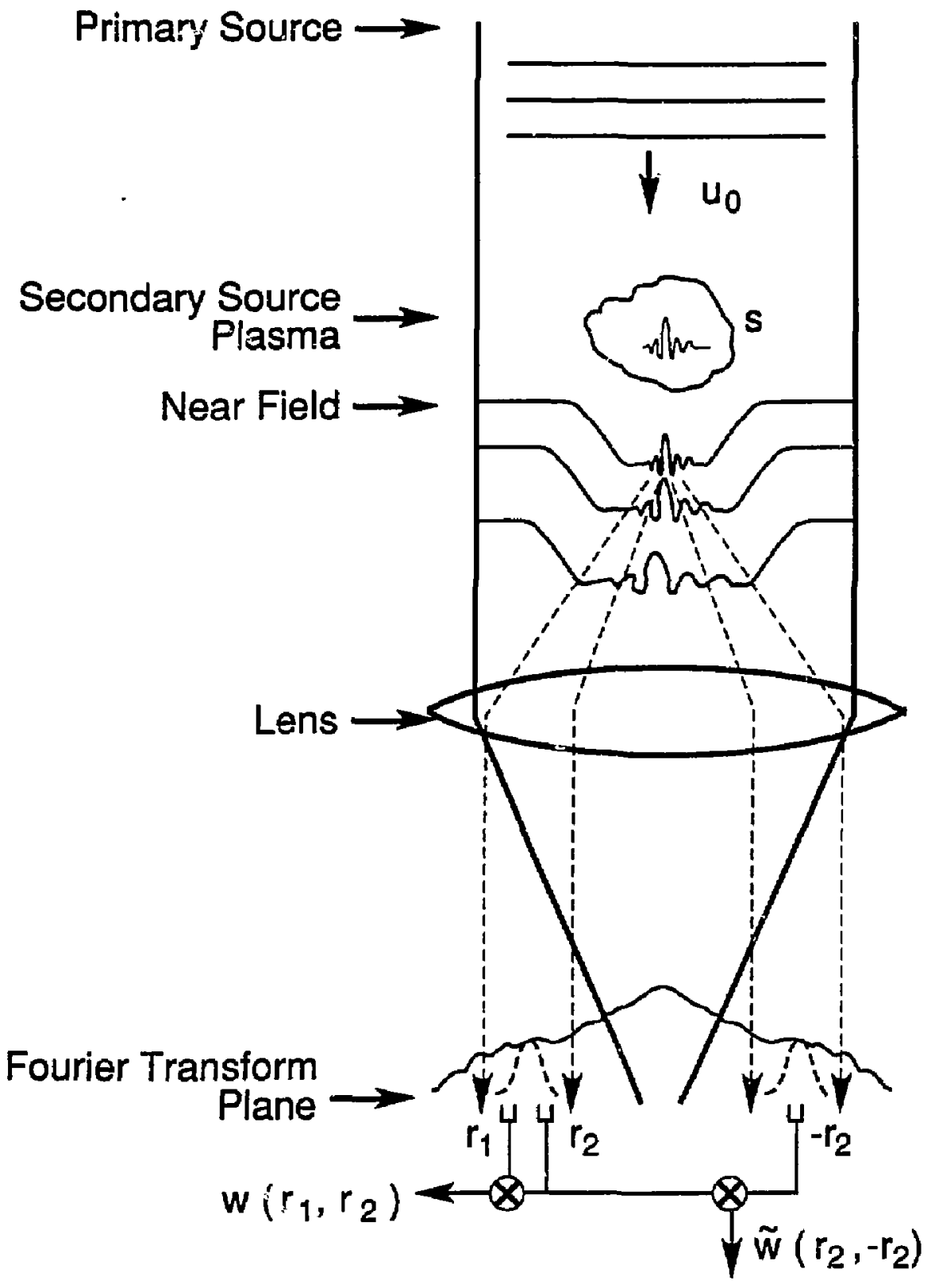

FIG. 1 